\title{
Elastography Evaluation of Benign Thyroid Nodules in Patients Affected by Hashimoto's Thyroiditis
}

\author{
Carlo Cappelli, Ilenia Pirola, Elena Gandossi, Annamaria Formenti, \\ Barbara Agosti, and Maurizio Castellano \\ Department of Clinical and Experimental Sciences, Endocrine and Metabolic Unit, University of Brescia, \\ Piazzale Spedali Civili No. 1, 25100 Brescia, Italy \\ Correspondence should be addressed to Carlo Cappelli; carlo.cappelli@unibs.it
}

Received 28 April 2015; Revised 25 June 2015; Accepted 28 June 2015

Academic Editor: Javier Salvador

Copyright (C) 2015 Carlo Cappelli et al. This is an open access article distributed under the Creative Commons Attribution License, which permits unrestricted use, distribution, and reproduction in any medium, provided the original work is properly cited.

The aim of the present prospective study was to evaluate the predictive value of elastography in benign thyroid nodules of patients affected by Hashimoto's thyroiditis (HT). From January 2011 to January 2012, 242 nodules in patients affected by HT were submitted to fine needle aspiration cytology (FNAC). All of the patients underwent sonography and elastography performed before FNAC. $230(95 \%)$ nodules were benign, 8 papillary cancers, and 4 follicular lesions. Score 1 was found in $79.1 \%$ of benign lesions (sensitivity 79.1\%; specificity 66.7\%; PPV 97.8\%; NPV 14.3\%; accuracy 78.5\%; $p<0.05$ ). In order to evaluate the outcome of thyroid ultrasound echogenicity in relation to elastography features of nodule(s), all the patients with benign nodules were stratified according to their hypoechoic pattern of thyroid (mild-moderate and severe). Following stratification score 1 was found in $84.2 \%$ of benign nodules (sensitivity 75.0\%; specificity $88.9 \%$; PPV 27.3\%; NPV 98.4\%; accuracy $88.2 \%$; $p<0.0001$ ) of patients with a mild-moderate ultrasound thyroid hypoechogenicity, whereas it was found in $60 \%$ of benign nodules $(p=0.715)$ of patients with a marked thyroid hypoechogenicity. Elastography appears to have limited value in detecting thyroid cancer in patients affected by Hashimoto's thyroiditis with severe hypoechoic thyroid tissue.

\section{Introduction}

Hashimoto's thyroiditis (HT), also known as chronic lymphocytic thyroiditis, is an autoimmune disease characterized by lymphocytic infiltration of the gland and production of autoantibodies that target thyroid peroxidase and/or thyroglobulin, resulting in tissue destruction and progressive loss of thyroid function [1].

After ultrasound (US) introduction into clinical practice in the late 1960s, thyroid ultrasonography proved to be very effective in the diagnostic approach to thyroid disorders [2-4]. Many studies have investigated whether the ultrasonographic characteristics of thyroid nodules are useful indicators of histological malignancy.

Overall, these investigations suggest a few ultrasonographic features that are significantly more frequent in malignant than in benign thyroid nodules, and some have tried to define a set of characteristics that identify nodules at higher risk of malignancy [5].
The characteristic US pattern of Hashimoto's glands consists of an array of tiny hypoechoic nodules that may become confluent, interspersed with echogenic fibrous bands [6]; nevertheless, US should be interpreted with caution due to the diffuse heterogeneity and the presence of pseudonodules related to ongoing inflammation. Elastography is a method which assesses the risk of the malignancy and provides information about the degree of hardness in tissue. Recently elastography has been proposed as a new technique for differentiating pseudonodules from nodules in HT [7] and benign from malignant lesions of thyroid gland [5, 8-10]. HT is able to change the hardness of the thyroid tissue. There have been few studies that have shown using elastography that $\mathrm{HT}$ is able to change the hardness of the thyroid gland decreasing its capacity to distinguish benign from malignant nodule $[11,12]$.

The aim of the present prospective study was to evaluate the predictive value of elastography in the characterization of benign thyroid nodules of patients affected by HT. 


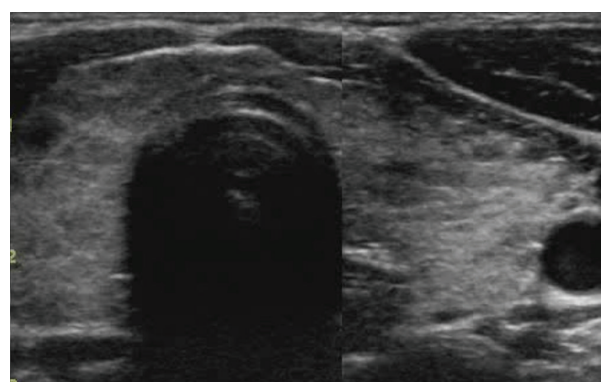

(a)

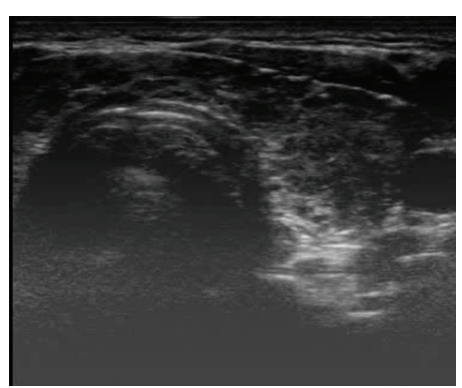

(b)

FIGURE 1: (a) Hashimoto's thyroiditis with mild sonographic hypoechogenicity. (b) Hashimoto's thyroiditis with severe sonographic hypoechogenicity.

\section{Materials and Methods}

From January 2011 to January 2012, 250 consecutive patients affected by HT with at least one solid nodule were submitted to fine needle aspiration cytology (FNAC). Cases were selected from patients referred to our thyroid unit for USguided FNAC. The selection criteria were as follows: (1) indication to FNAC in accordance with the major guidelines $[5,14]$; (2) positive test for thyroglobulin antibody (TgAb) and/or thyroid peroxidase antibody (TPOAb) and a thyroid gland with hypoechoic pattern at US evaluation.

All of the patients underwent sonography and elastography performed by the same skilled sonographer (CC) before US-guided FNAC. Thyroid sonography and elastography were performed with a real-time instrument (Vision 900; Hitachi Medical System, Tokyo, Japan) equipped with a linear probe with a central frequency of 6-13 $\mathrm{MHz}$.

The sonographic hypoechogenicity of thyroid gland (Figures 1(a) and 1(b)) was classified from mild-moderate to severe in accordance with literature [15-18].

Findings at elastography were classified according to the elasticity scores by Rago et al. [13]. Briefly, score 1 indicated nodules with high elasticity, score 2 nodules with intermediate elasticity, and score 3 nodules with low elasticity (Table 1). To minimize the intraobserver variability, the freehand compression applied on the neck was standardized by real-time measurement displayed on a numeric scale (graded 1-5) to maintain an intermediated level optimal for elastographic evaluation $[6,8]$.

The serum concentrations of $\mathrm{TgAb}$ (normal range: $<60 \mathrm{U} / \mathrm{mL}$ ) and TPOAb (normal range: $<60 \mathrm{U} / \mathrm{mL}$ ) were measured using immunochemiluminescent assays employing commercial kits (Brahms, Hennigsdorf, Germany).

All patients gave their informed consent to participate in the study, which was performed in accordance with the Declaration of Helsinki.

All data were analyzed with SPSS version 17 software (SPSS Inc., Chicago, IL). Comparisons between groups were performed by an analysis of variance general linear model; a $\chi^{2}$ test was used for categorical variables. Statistical significance was considered at $p<0.05$.
TABLE 1: The applied standard elastography color scoring system according to Rago criteria [13]. A score of 1 indicates even elasticity in the whole nodule, score 2 elasticity only at the peripheral part of the nodule, and score 3 no elasticity in the nodule and in the posterior shadowing.

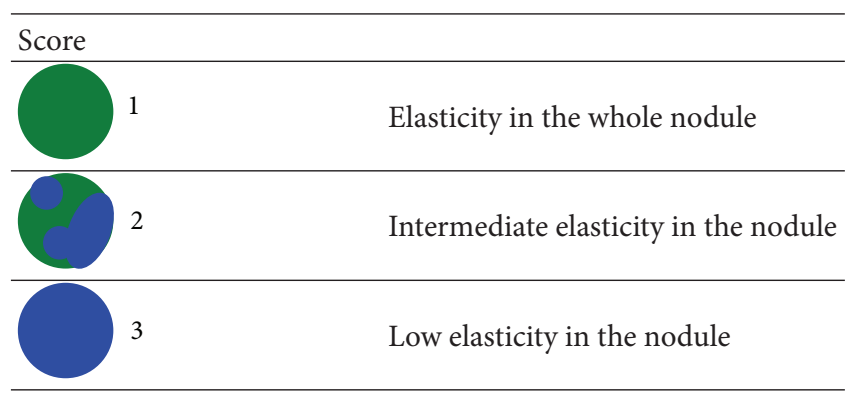

TABLE 2: Elasticity score of the 242 nodules submitted to cytology that resulted in benign nodules or carcinoma.

\begin{tabular}{lccc}
\hline Elasticity score & Benign $(n)$ & Cancer $(n)$ & Tot. $(n)$ \\
\hline Score 1 & 182 & 2 & 184 \\
Score 2 & 39 & 5 & 44 \\
Score 3 & 9 & 5 & 14 \\
\hline
\end{tabular}

\section{Results}

Two hundred fifty consecutive patients (202 female, 48 male), mean age $52.2 \pm 11.2$ years, were submitted to US-guided FNAC.

Of the 262 nodules submitted to cytology, 20 (7.6\%) appeared to be nondiagnostic, and as a consequence 16 patients were excluded from the study. Among 234 patients two hundred forty-two nodules were collected: 230 (95\%) were benign, 8 papillary cancers, and 4 follicular lesions. Histology confirmed 8 papillary and 4 follicular cancers.

The lesions classified as scores 1,2, and 3 on elastography are listed in Table 2.

Score 1 was found in 182/230 (79.1\%) of benign lesions (sensitivity $79.1 \%$; specificity $66.7 \%$; positive predictive value 


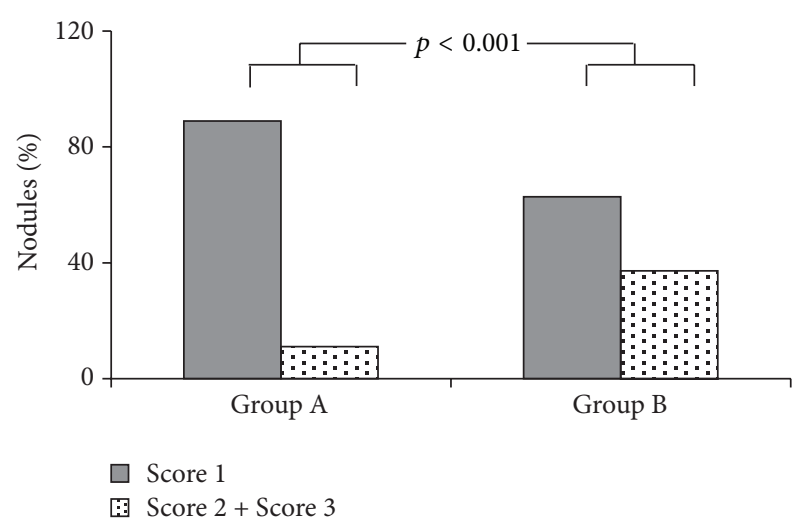

FIGURE 2: Elasticity score in nodules with benign diagnostic cytology in relation to thyroid hypoechogenicity pattern.

97.8\%; negative predictive value $14.3 \%$; accuracy $78.5 \%$; $p<$ $0.05)$.

All 227 patients with benign nodules were stratified in accordance with the echogenic pattern of thyroid gland (mild-moderate/severe): 128 (56\%) patients presented a mildmoderate thyroid echogenicity (Group A) and 99 (44\%) a severe one (Group B). The two groups were then stratified in accordance with the elastographic scoring of the nodule (scores 1,2, and 3). The two groups were superimposable for gender $(86 / 42$ versus $64 / 35 \mathrm{~F} / \mathrm{M})$, age $(53.1 \pm 12.8$ versus 49.6 \pm 10 , years), and TSH values ( $3.9 \pm 1.1$ versus $4.0 \pm 0.9 \mathrm{mU} / \mathrm{L})$.

Following stratification, a significant different behavior emerged in the two groups (Figure 2). In detail, score 1 was found in $84.2 \%$ of benign nodules of patients with a mildmoderate ultrasound thyroid hypoechogenicity (sensitivity $75.0 \%$; specificity $88.9 \%$; positive predictive value $27.3 \%$; negative predictive value $98.4 \%$; accuracy $88.2 \%$; $p<0.0001$ ) and in $60 \%$ of patients with a marked thyroid hypoechogenicity (sensitivity $50.0 \%$; specificity $62.8 \%$; positive predictive value $5.9 \%$; negative predictive value $96.2 \%$; accuracy $62.2 \%$; $p=$ $0.715)$.

\section{Discussion}

As described by Gutekunst et al. thirty years ago HT is associated with a diffuse reduction in thyroid echo levels (hypoechogenicity) [19], with a correlation between serum TPOAb levels and the degree of hypoechogenicity $[18,20]$, that have been classified from mild-moderate to severe [17, 18]. After US introduction into clinical practice in the late nineteen sixties thyroid ultrasonography proved to be very effective in the diagnostic approach to thyroid disease [21].

Elastography has been proposed as a new and promising technique for thyroid nodules evaluation $[8-10,13]$. The first evidence suggested that interobserver agreement is not reliable in the diagnosis of malignant lesions but recent data clearly shows a good interobserver and intraobserver reproducibility in detecting thyroid cancer $[22,23]$.

Magri et al. evidenced that shear wave elastography correctly defined the elasticity of thyroid nodules independently of the coexistence of autoimmune thyroiditis, being able to differentiate nodular tissue from the surrounding parenchyma [6]. The results of the present study confirm that elastography is effective in distinguishing between benign and malignant nodules in patients affected by HT, showing however a lack of accuracy in patients affected by HT presenting diffuse marked hypoechoic thyroid echo-structure (i.e., severe sonographic thyroid hypoechogenicity). On the contrary, two recent studies have shown that strain index reflects malignancy better than the elastoscore in HT patients $[11,12]$.

Taking into account the elastography performance in our patients without stratification by sonographic hypoechogenicity, our results also showed a lack of accuracy, but for the first time, we showed that elastography has a high performance when the thyroid echogenicity is not severe.

As already reported by Pedersen et al. [24] stronger inflammatory process has been shown in severe sonographic thyroid hypoechogenicity due to the diffuse infiltration of the thyroid parenchyma with lymphocytes and fibrosis [20, 25]. It is conceivable that a benign process, likely represented by nodular fibrosis, is responsible for the hard elastographic pattern of many thyroid nodules in patients with HT. In fact, histologically proven fibrotic nodules of the thyroid appear to be characterized by a high stiffness index at elastographic evaluation [26] and several hard nodules were detected in patients previously submitted to radioiodine treatment which is known to induce fibrosis [27]. Taking into account these studies, we have hypothesized that higher inflammatory process, lymphocytic infiltration, and fibrosis characterize HT with severe sonographic thyroid hypoechogenicity, but these reactions are also present in benign nodule(s) therefore reducing the accuracy of elastographic evaluation in these patients. Our results are in accordance with those of Scacchi et al. obtained in acromegalic patients, who showed that higher fibrosis developed in acromegalic thyroid patients justifying the worst performance of elastography in detecting cancer [28].

The present study has few limitations; firstly no histological data of benign nodules are given and secondly there is the relative small size of patients enrolled in the study. Another potential confounding factor may be the possibility that "benign nodules" were represented by pseudonodular fibrotic infiltration.

Unfortunately, we do not have histological data about cytologically proven benign nodule(s). Only a small number of patients $(n=26)$ with large benign nodules were submitted to thyroidectomy and in all these patients histology confirmed the benign nature of the lesions (data not shown). However, we must underline the accuracy in terms of sensitivity, specificity, and false negative and positive rate of FNAC $[29,30]$ to consider this procedure the gold-standard in differentiating benign from malignant thyroid nodules in many guidelines $[5,14]$. Moreover, not only cytology $[29,30]$ but also elastography $[13,26]$ well distinguishes true nodules from pseudonodular ones. For these reasons we believe that the lack of histological data in our study cannot diminish the value of our results. Prospective large studies are needed to confirm our observation. 
In conclusion our study has demonstrated a high prevalence of hard thyroid nodule at elastography evaluation in patients with HT. Interestingly this is particularly evident when the hypoechoic pattern of thyroid gland is severe, suggesting an extensive infiltration of the thyroid parenchyma with lymphocytes and fibrosis. The hypothesis that nodular fibrosis might account for this elastography pattern is conceivable but needs histopathological confirmation. Elastography evidences a lack of accuracy when it is performed in patients affected by HT presenting diffuse severe hypoechoic thyroid echo-structure.

\section{Conflict of Interests}

No potential conflict of interests relevant to this paper was reported.

\section{Acknowledgment}

The authors are thankful to Ms. Courtney M Cliffe for English support.

\section{References}

[1] C. M. Dayan and G. H. Daniels, "Chronic autoimmune thyroiditis," The New England Journal of Medicine, vol. 335, no. 2, pp. 99-107, 1996.

[2] A. S. Alzahrani, G. Ceresini, and S. A. Aldasouqi, "Role of ultrasonography in the differential diagnosis of thyrotoxicosis: a noninvasive, cost-effective, and widely available but underutilized diagnostic tool," Endocrine Practice, vol. 18, no. 4, pp. 567578, 2012.

[3] W.-J. Moon, J. H. Baek, S. L. Jung et al., "Ultrasonography and the ultrasound-based management of thyroid nodules: consensus statement and recommendations," Korean Journal of Radiology, vol. 12, no. 1, pp. 1-14, 2011.

[4] G. T. Kangelaris, T. B. Kim, and L. A. Orloff, "Role of ultrasound in thyroid disorders," Otolaryngologic Clinics of North America, vol. 43, no. 6, pp. 1209-1227, 2010.

[5] H. Gharib, E. Papini, R. Paschke et al., "American Association of Clinical Endocrinologists, Associazione Medici Endocrinologi, and European Thyroid Association medical guidelines for clinical practice for the diagnosis and management of thyroid nodules: executive summary of recommendations," Endocrine Practice, vol. 16, no. 3, pp. 468-475, 2010.

[6] F. Magri, S. Chytiris, V. Capelli et al., "Shear wave elastography in the diagnosis of thyroid nodules: feasibility in the case of coexistent chronic autoimmune Hashimoto's thyroiditis," Clinical Endocrinology, vol. 76, no. 1, pp. 137-141, 2012.

[7] D. Yildirim, B. Gurses, B. Gurpinar, B. Ekci, B. Colakoglu, and A. Kaur, "Nodule or pseudonodule? differentiation in Hashimoto's thyroiditis with sonoelastography," Journal of International Medical Research, vol. 39, no. 6, pp. 2360-2369, 2011.

[8] C. Cappelli, I. Pirola, E. Gandossi et al., "Real-time elastography: a useful tool for predicting malignancy in thyroid nodules with nondiagnostic cytologic findings," Journal of Ultrasound in Medicine, vol. 31, no. 11, pp. 1777-1782, 2012.

[9] J. Ding, H. Cheng, C. Ning, J. Huang, and Y. Zhang, "Quantitative measurement for thyroid cancer characterization based on elastography," Journal of Ultrasound in Medicine, vol. 30, no. 9, pp. 1259-1266, 2011.

[10] P. Xing, L. Wu, C. Zhang, S. Li, C. Liu, and C. Wu, "Differentiation of benign from malignant thyroid lesions: calculation of the strain ratio on thyroid sonoelastography," Journal of Ultrasound in Medicine, vol. 30, no. 5, pp. 663-669, 2011.

[11] B.-J. Liu, H.-X. Xu, Y.-F. Zhang et al., "Acoustic radiation force impulse elastography for differentiation of benign and malignant thyroid nodules with concurrent Hashimoto's thyroiditis," Medical Oncology, vol. 32, no. 3, article 50, 2015.

[12] M. Şahin, E. Çakal, M. Özbek et al., "Elastography in the differential diagnosis of thyroid nodules in Hashimoto thyroiditis," Medical Oncology, vol. 31, no. 8, article 97, 2014.

[13] T. Rago, M. Scutari, F. Santini et al., "Real-time elastosonography: useful tool for refining the presurgical diagnosis in thyroid nodules with indeterminate or nondiagnostic cytology," Journal of Clinical Endocrinology and Metabolism, vol. 95, no. 12, pp. 5274-5280, 2010.

[14] D. S. Cooper, G. M. Doherty, B. R. Haugen et al., "Revised American thyroid association management guidelines for patients with thyroid nodules and differentiated thyroid cancer," Thyroid, vol. 19, no. 11, pp. 1167-1214, 2009.

[15] T. Rago, L. Chiovato, L. Grasso, A. Pinchera, and P. Vitti, “Thyroid ultrasonography as a tool for detecting thyroid autoimmune diseases and predicting thyroid dysfunction in apparently healthy subjects," Journal of Endocrinological Investigation, vol. 24, no. 10, pp. 763-769, 2001.

[16] P. Vitti and T. Rago, "Thyroid ultrasound as a predicator of thyroid disease," Journal of Endocrinological Investigation, vol. 26, no. 7, pp. 686-689, 2003.

[17] P. Vitti, T. Rago, S. Mazzeo et al., "Thyroid blood flow evaluation by color-flow doppler sonography distinguishes Graves' disease from Hashimoto's thyroiditis," Journal of Endocrinological Investigation, vol. 18, no. 11, pp. 857-861, 1995.

[18] U. Schiemann, W. Avenhaus, J. W. Konturek, R. Gellner, K. Hengst, and M. Gross, "Relationship of clinical features and laboratory parameters to thyroid echogenicity measured by standardized grey scale ultrasonography in patients with Hashimoto's thyroiditis," Medical Science Monitor, vol. 9, no. 4, pp. 13-17, 2003.

[19] R. Gutekunst, W. Hafermann, T. Mansky, and P. C. Criba, "Ultrasonography related to clinical and laboratory findings in lymphocytic thyroiditis," Acta Endocrinologica, vol. 121, no. 1, pp. 129-135, 1989.

[20] C. Marcocci, P. Vitti, F. Cetani, F. Catalano, R. Concetti, and A. Pinchera, "Thyroid ultrasonography helps to identify patients with diffuse lymphocytic thyroiditis who are prone to develop hypothyroidism," The Journal of Clinical Endocrinology \& Metabolism, vol. 72, no. 1, pp. 209-213, 1991.

[21] C. Cappelli, I. Pirola, E. De Martino et al., "The role of imaging in Graves' disease: a cost-effectiveness analysis," European Journal of Radiology, vol. 65, no. 1, pp. 99-103, 2008.

[22] F. Ragazzoni, M. Deandrea, A. Mormile et al., "High diagnostic accuracy and interobserver reliability of real-time elastography in the evaluation of thyroid nodules," Ultrasound in Medicine and Biology, vol. 38, no. 7, pp. 1154-1162, 2012.

[23] D.-J. Lim, S. Luo, M.-H. Kim, S.-H. Ko, and Y. Kim, "Interobserver agreement and intraobserver reproducibility in thyroid ultrasound elastography," The American Journal of Roentgenology, vol. 198, no. 4, pp. 896-901, 2012.

[24] O. M. Pedersen, N. P. Aardal, T. B. Larssen, J. E. Varhaug, O. Myking, and H. Vik-Mo, "The value of ultrasonography in 
predicting autoimmune thyroid disease," Thyroid, vol. 10, no. 3, pp. 251-259, 2000.

[25] H.-C. Yeh, W. Futterweit, and P. Gilbert, "Micronodulation: ultrasonographic sign of hashimoto thyroiditis," Journal of Ultrasound in Medicine, vol. 15, no. 12, pp. 813-819, 1996.

[26] M. Dighe, U. Bae, M. L. Richardson, T. J. Dubinsky, S. Minoshima, and Y. Kim, "Differential diagnosis of thyroid nodules with US elastography using carotid artery pulsation," Radiology, vol. 248, no. 2, pp. 662-669, 2008.

[27] W.-J. Shih, B. Mitchell, and J. C. Schott, "Scarred atrophic thyroid after I-131 theraphy for Graves' disease documented at autopsy," Journal of the National Medical Association, vol. 94, no. 10, pp. 915-919, 2002.

[28] M. Scacchi, M. Andrioli, C. Carzaniga et al., "Elastosonographic evaluation of thyroid nodules in acromegaly," European Journal of Endocrinology, vol. 161, no. 4, pp. 607-613, 2009.

[29] D. W. Kim, H. J. Choo, J. S. Park et al., "Ultrasonographyguided fine-needle aspiration cytology for thyroid nodules: an emphasis on one-sampling and biopsy techniques," Diagnostic Cytopathology, vol. 40, no. 1, pp. E48-E54, 2012.

[30] K. Sugino, K. Ito, M. Nagahama et al., "Diagnostic accuracy of fine needle aspiration biopsy cytology and ultrasonography in patients with thyroid nodules diagnosed as benign or indeterminate before thyroidectomy," Endocrine Journal, vol. 60, no. 3, pp. 375-382, 2013. 


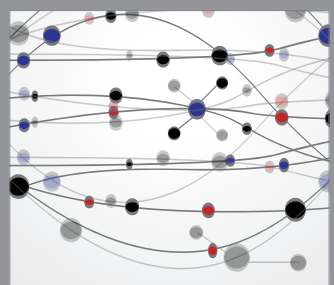

The Scientific World Journal
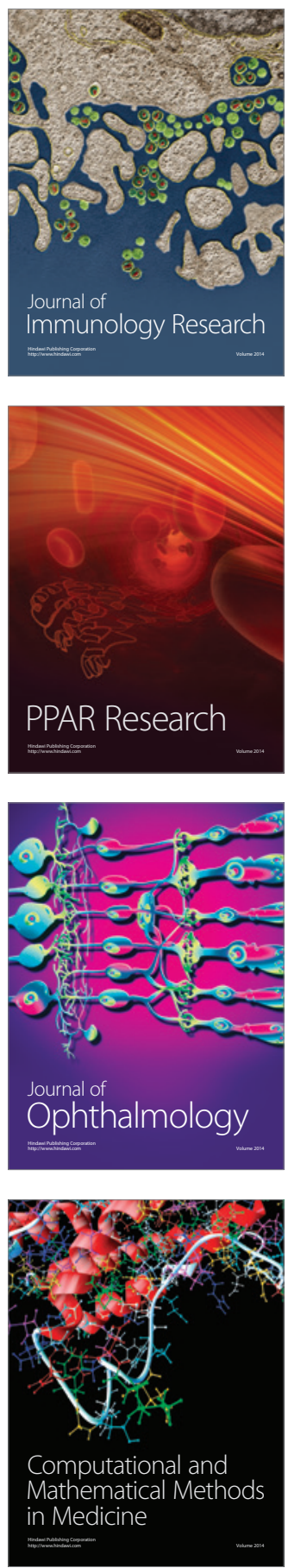

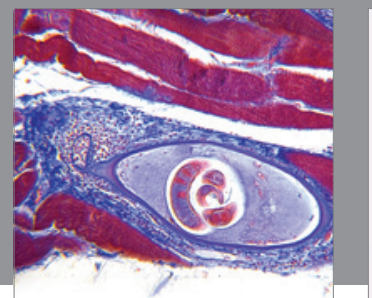

Gastroenterology

Research and Practice
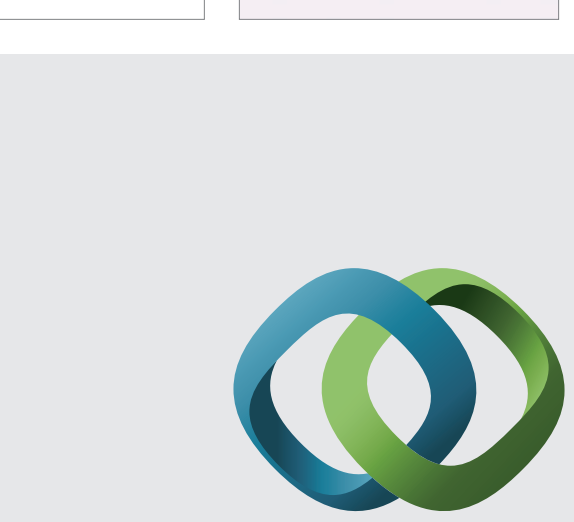

\section{Hindawi}

Submit your manuscripts at

http://www.hindawi.com
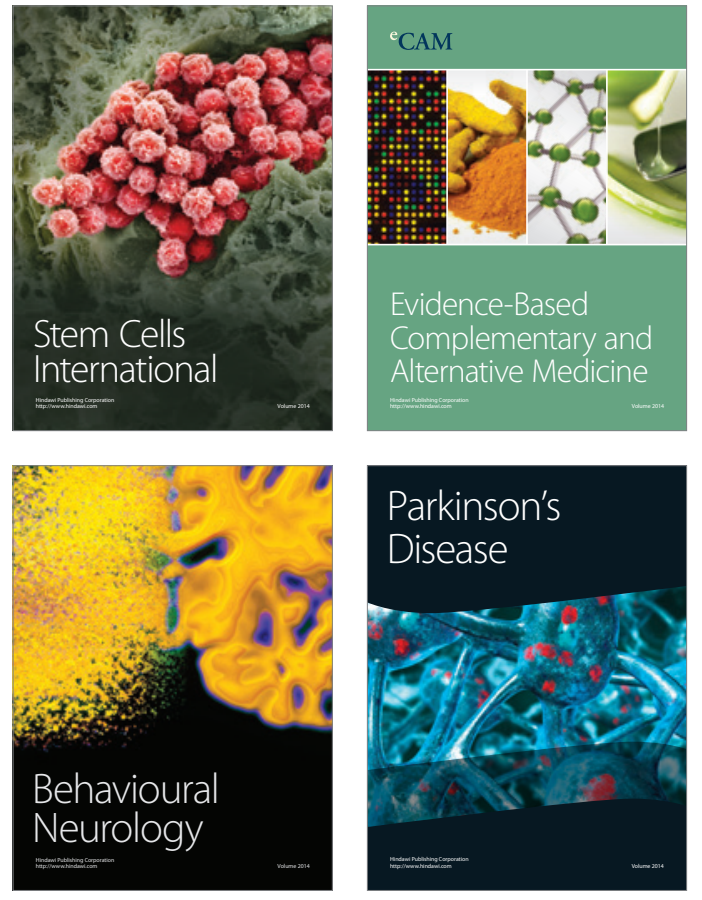
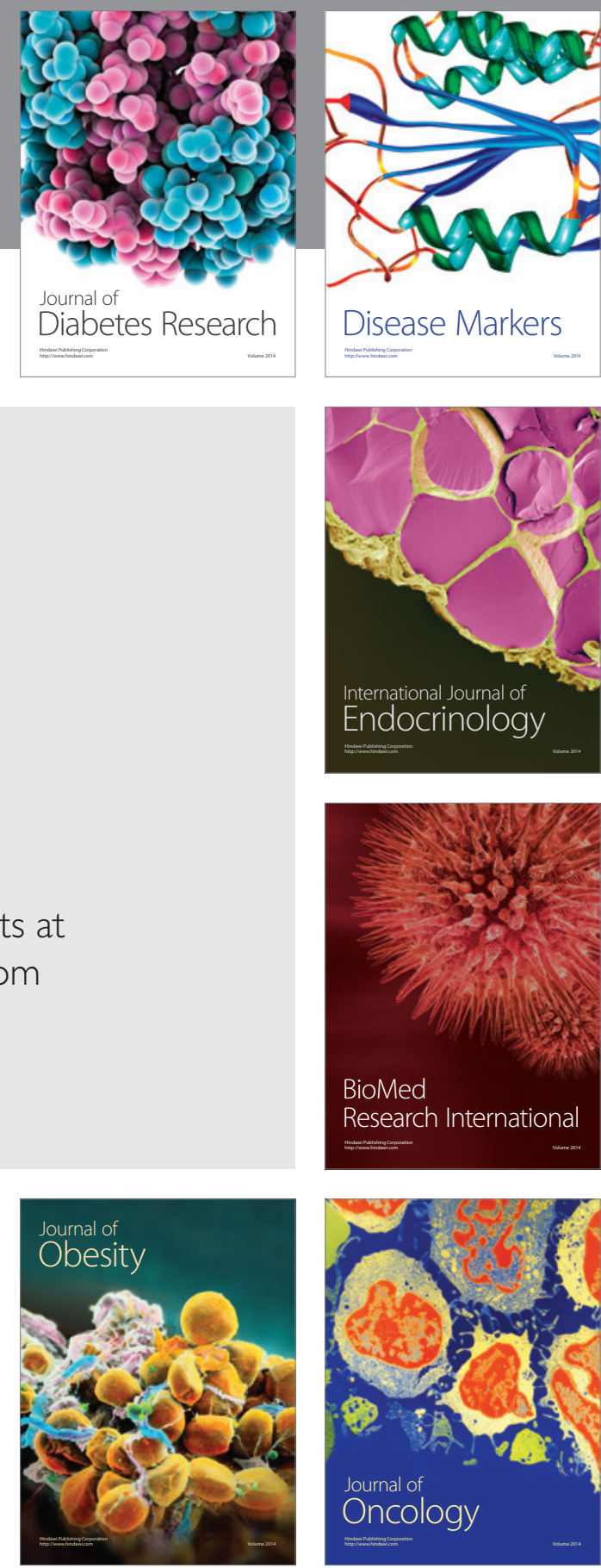

Disease Markers
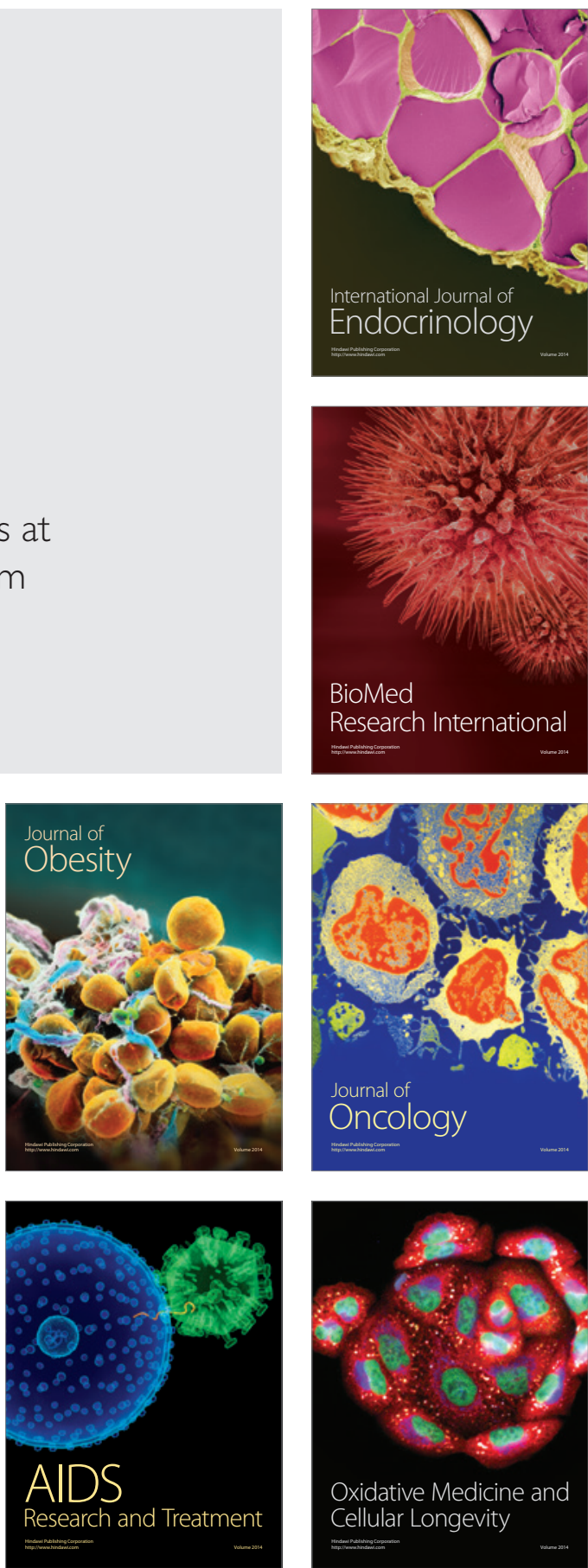